Aksaray University
Journal of Science and Engineering
e-ISSN: 2587-1277
http://dergipark.gov.tr/asujse
http://asujse.aksaray.edu.tr

Research Article

\title{
The Effect of The CAD Data Exchange on the Motion Analysis in the Solidworks CAD Program
}

\author{
Mustafa $\mathrm{Er}^{1}$, Yunus Kayir ${ }^{2, * *}$ \\ ${ }^{1}$ Gazi University Graduate School of Natural and Applied Sciences, 06500, Ankara, Turkey \\ ${ }^{2 *}$ Gazi University, Technology Faculty, Department of Manufacturing Engineering, 06500, Ankara, Turkey, \\ -Received Date: 15 Mar $2019 \quad$ •Revised Date: 3 Apr $2019 \quad$ •Accepted Date: 27 May $2019 \quad$ •Published Online: 29 June 2019
}

\begin{abstract}
Today, computer-aided systems are widely used in design and manufacturing. There are many CAD and CAM programs in the world with different features developed for this purpose. Common file formats are developed in order to make data exchange between CAD and CAM programs. However, losses can occur in data exchange. In this study, it was thought that a 3D model obtained in a CAD program would have an effect on the results obtained if another CAD was used for any analysis. For this purpose, a crank-rod mechanism is designed as a 3D in a common CAD program. The parts used in the crank-rod mechanism are modeled as solid. The parts were save in IGES, STEP and SLDPRT common file formats. Assemblies were realized separately for 3 different CAD formats. The motion analysis was performed on the crank-rod mechanisms and the results were compared. The results of the analysis showed that there were differences between SLDPRT, STEP and IGES data of the crank-rod mechanisms.
\end{abstract}

\section{Keywords}

Motion Analysis, IGES, STEP, Assembly

*Corresponding Author: Yunus Kayir, ykayir@gazi.edu.tr 


Aksaray University
Journal of Science and Engineering
e-ISSN: 2587-1277
http://dergipark.gov.tr/asujse
$\underline{\text { http://asujse.aksaray.edu.tr }}$

Research Article

\title{
Solidworks Programında Yapılan Hareket Analizine CAD Veri Değişiminin Etkisi
}

\author{
Mustafa Er ${ }^{1}$, Yunus Kayır ${ }^{2 * *}$ \\ ${ }^{1}$ Gazi Üniversitesi Fen Bilimleri Enstitüsü, 06500,Ankara, mustafa.er2@gazi.edu.tr \\ ${ }^{2}$ Gazi Üniversitesi, Teknoloji Fakültesi, Imalat Mühendisliği, Ankara,06500,Türkiye, ykayir@gazi.edu.tr \\ -Gönderi Tarihi: 15 Mart $2019 \quad$-Revizyon Tarihi: 3 Nisan $2019 \quad \cdots \quad$-Kabul Tarihi: 27 Mayıs $2019 \quad$-C Cevrimiçi Yayln Tarihi: 29 Haz 2019
}

\section{Özet}

Günümüzde, tasarım ve imalat işlerinde bilgisayar destekli sistemler yaygın olarak kullanılmaktadır. Dünyada, bu amaçla geliştirilmiş farklı özelliklere sahip birçok BDT ve BDİ programları bulunmaktadır. BDT ve BDİ programlarının birbirleri arasında veri alışverişinin yapılabilmesinde ise ortak dosya formatları geliştirilmiştir. Fakat yapılan veri alışverişlerinde ise kayıplar meydana gelebilmektedir. Bu çalışmada, bir BDT programında elde edilen bir modelin, herhangi bir analiz için başka bir BDT kullanılması durumunda elde edilecek olan sonuçlar üzerinde etkili olacağı düşünülmüştür. Bu amaçla, yaygın kullanıma sahip bir BDT programında krank-biyel mekanizması tasarlanmıştır. Krank-biyel mekanizmasında kullanılan parçalar katı olarak modellenmiştir. Modellenen parçalar, IGES, STEP ve SLDPRT dosya formatlarında kaydedilmiştir. 3 farklı format için ayrı ayrı montajlar gerçekleştirilmiştir. Elde edilen krank-biyel mekanizmaları üzerinde hareket analizleri yapılmış ve sonuçlar karşılaştırılmıştır. Analiz sonuçları, SLDPRT, STEP ve IGES dosya formatlarında kaydedilen mekanizmalara ait veriler arasında farklılıkların bulunduğunu göstermiştir.

\section{Anahtar Kelimeler}

Hareket Analizi, IGES, STEP, Montaj

\footnotetext{
*Sorumlu Yazar: Yunus Kayır, ykayir@gazi.edu.tr
} 


\section{GİRIS}

BDT (Bilgisayar Destekli Tasarım) ve BDİ (Bilgisayar Destekli İmalat) sistemlerin birlikteliği günümüzde önemli bir araştırma konusu olup bu problemin çözümüne yönelik çok sayıda çalışma yapılmaktadır. Her bir BDT sistemi bir parçanın geometrik modeli için kendi iç temsil tekniklerini ve veri yapısını kullanmaktadır. Bu da farklı BDT ve BDİ sistemleri arasında veri iletişiminin sağlanamaması anlamına gelmektedir. Bundan dolayı, BDT ve BDİ sistemleri arasında veri iletişimini sağlayacak bir ara yüz gereksinimi ortaya çıkmıştır. Bu gereksinimi karşılamak amacıyla özellikle 1980' li yıllarda tasarım ve imalatta standardizasyon çalışmaları başlatılmıştır. Günümüzde DXF, IGES, SET, VDA-FS, CAD*I, STEP gibi geliştirilen grafik standartları algoritma geliştirmede ve veri dönüşümünde halen yaygın olarak kullanılmaktadır [1, 2]. Grafik Değişim Özelliği (IGES: Initial Graphics Exchange Specification) ve Ürün Verilerinin Değişimi için Standart (STEP: Standard for The Exchange of Product model data), en yaygın kullanılan iki nötr dosya formatıdır. Bu formatlar, farklı BDT ve BDİ sistemlerinin birbirleri ile veri paylaşımını sağlar. IGES, elektrik, tasarım, mekanik gibi çok çeşitli uygulama alanlarında elde edilen verileri bir BDT yazılımından diğerine rahatlıkla aktarılabilmesinde kullanılan standart bir formattır [3]. IGES BDT/BDİ sistemlerinin veri aktarımında yaygın olarak kullanılan bir standarttır [4]. IGES, endüstriden akademik çalışmalara kadar birçok kullanım alanına sahiptir. Günümüzde piyasaya çıkan birçok BDT/BDİ sistemi IGES standardını kullanmaktadır. IGES standardında bilgilerin çıkarılmasında; ASCII ve ikili sayı (binary) formatı olmak üzere iki tip dosya yapısı desteklenmektedir. ASCII dosya yapısında bilgiler 80 karakterden oluşan satırlarla ifade edilirken ikili dosya yapısında ise bilgiler ikili sayı sistemine göre kodlanmaktadır [5]. IGES, 1980 den beri ANSI standardı olarak geliştirilmiştir.

STEP ise, IGES alt yapısı ile geliştirilen bir standart olup, özellikle de otomobil, havacılık ve savunma sektörlerinin desteği nedeniyle tüm BDT ve BDİ sistemlerinde kullanımı büyük önem kazanmıştır [6]. STEP, verilerinin karşılıklı alışverişi için gerekli mekanizmaların ve tanımların yanı sıra, ürüne yönelik bazı bilgileri de kayıt altına almaktadır. Ürünün yaşam döngüsü olarak bilinen değişim, tasarım, üretim, inceleme, bakım ve elden çıkarma gibi bilgiler STEP formatı ile tutulmaktadır. Bu işlemler sırasında bir ürün hakkında oluşturulan bilgiler birçok amaç için kullanılır. STEP, bir ürünün ve bileşenlerinin tasarım, üretim, pazarlama ve tedarikine katkıda bulunan, bilgiye erişen ve bilgi paylaşan tüm araçlar için bulunur [2]. STEP'in 1.0 versiyonu 1988' de yayınlanmış olup, ISO tarafından grafik verileri standardı olarak kabul edilmiştir. 
STEP, bir ürünün tanımlanması için gerekli olan geometri, topoloji, şekil özelliği, tolerans, yüzey pürüzlülüğü ve malzeme gibi bilgileri içermektedir [7].

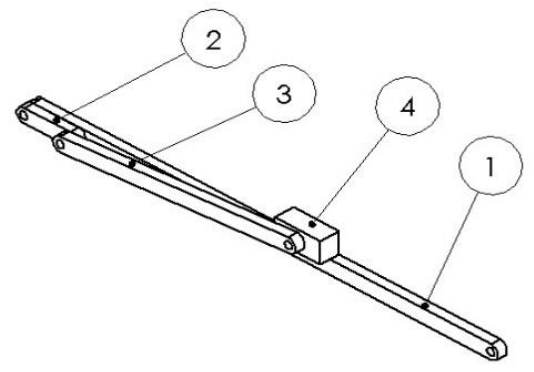

Şekil 1. Krank biyel mekanizmas1

Yaygın kullanılan BDT sistemleri SOLIDWORKS, UGS, Pro / E ve CATIA arasında veri alışverişi nötr (tarafsız) olarak desteklenmektedir. Ancak, kullanılan BDT programında oluşturulan modele ait bazı özellikler (örneğin montaj eşleşme şartları) IGES ve STEP olarak dışarı aktarılmasında dikkate alınmamaktadır [8]. Literatür çalışmalarından anlaşılacağı gibi IGES ve STEP, farklı BDT ve BDİ programları arasında veri alışverişinde sıklıkla kullanılmaktadır. Bu çalışmada, SOLIDWORKS tasarım programında 3B katı modellenen parçalar ile oluşturulan basit bir krank-biyel mekanizması üzerinde hareket analizleri yapılmıştır. Krank-biyel mekanizmasında kullanılan 3B parçaların IGES ve STEP dosyası olarak dışarıdan alınması durumunda yapılacak olan hareket analizi sonuçları üzerindeki olası değişimler incelenmiştir

\section{MALZEME VE YÖNTEMLER}

$\mathrm{Bu}$ çalışmada, bir CAD programında hareket analizleri yapılmıştır. Hareket analizleri için basit bir mekanizma modellenmiştir.

Tablo 1. Krank-biyel mekanizmasında kullanılan parçaların teknik resmi

\begin{tabular}{|c|c|c|}
\hline Parça No & Montaj Parçaların Teknik Resimleri Ölçüler (mm) & Kalınlık (mm) \\
\hline 1 & 500 & 10 \\
\hline 2 & $(-1) \cdot{ }^{R 8}$ & 10 \\
\hline 3 & (6). & 10 \\
\hline 4 & 50 & 20 \\
\hline
\end{tabular}




\subsection{Krank-Biyel Mekanizması}

Krank-biyel mekanizması, makine tasarımında yoğun bir şekilde kullanılan bir mekanizmadır. Genel olarak bir dönme hareketini bir öteleme hareketine çevirmek için kullanıldığı gibi bir öteleme hareketini dönme hareketine çevirmek içinde kullanılabilir [9]. Şekil 1'de krank-biyel mekanizmasının parçaları gösterilmiştir. Tablo 1'de krank-biyel mekanizmasında kullanılan 3B parçaların teknik resim ölçüleri verilmiştir.

\subsection{SOLIDWORKS CAD Programı}

Hareket analizleri SOLIDWORKS programında yapılmıştır. SOLIDWORKS iki ve üç boyutlu bilgisayar destekli tasarım için kullanılan bir BDT programıdır. SOLIDWORKS günümüzde hem 2B hem de 3B çizimler için etkin olarak kullanılmaktadır. Resmi verilere göre dünya üzerinde aktif olarak kullanılan SOLIDWORKS programı en geniş kullanıma sahip olan BDT programlarının başında gelir. Pek çok güncel BDT programı gibi parametrik özellik tabanlı modelleme teknolojisine sahip olan SOLIDWORKS ile katı modeller parametrik yaklaşımlarla oluşturularak basit ve kolay düzenlenebilir bir yapı elde edilir. SOLIDWORKS programında hazırlanan parça modelleri birleştirilerek kolaylıkla ürün montaj1 yapılabilir [10]. SOLIDWORKS analiz ve animasyon özelliğine de sahiptir. Bu çalışmada krank-biyel mekanizmasının tasarımı ve analizlerinde SOLIDWORKS programının 2015 sürümü kullanılmıştır.

\subsection{Hareket Analizinde Kullanılan Parametreler}

$\mathrm{Bu}$ çalışmada, krank-biyel mekanizmasında kullanılacak olan parçalar (Tablo 1) 3B olarak modellenmiştir. Daha sonra hareket analizleri için bu parçalar montaj edilmiştir. Devamında tasarlanan mekanizma üç farklı dosya formatında, STEP, IGES ve SOLIDWORKS programının kendi dosya uzantısı olan SLDPRT kaydedilmiştir. Kaydedilen tüm dosyalar SOLIDWORKS montaj ortamında açılmıştır. Fakat açılan STEP ve IGES dosya ile montaj bileşenlerinin tanımlanmadığı görülmüştür. Diğer bir deyişle, montaj ortamında açılan krankbiyel mekanizmasını oluşturan parçaların birbirleri ile ayrı olduğu bir montaj ilişkisine sahip olmadığ1 görülmüştür. Bunun üzerine parçaların tekrar montajı yapılarak kaydedilmiştir. Hareket analizleri öncesinde tüm montaj parçaların her birine malzeme olarak AISI 1020 çeliği tanımlanmıştır (Şekil 2). Yapılan malzeme ataması ile krank-biyel mekanizmasının (SLDPRT dosyası) elde edilen fiziksel özelliklerinden bazıları Tablo 2'de verilmiştir. Mekanizmayı hareket ettirecek olan motorun dönüş hızı için 7,5 rpm seçilmiştir. Yerçekimi dikkate alınmıştır. Bu parametreler uygulandıktan sonra hareket analizi yapılmıştır. 
Tablo 2. Krank-biyel mekanizmasının fiziksel özellikleri

\begin{tabular}{|c|r|r|r|r|r|}
\hline & Parça 1 & Parça 2 & Parça 3 & Parça 4 & Montaj \\
\hline Kütle $(\mathrm{g})$ & 639,94 & 71,14 & 323,94 & 181,66 & 1216,68 \\
\hline Hacim $\left(\mathrm{mm}^{3}\right)$ & 81005,31 & 9005,31 & 41005,31 & 22994,69 & 154010,62 \\
\hline Yüzey Alan1 $\left(\mathrm{mm}^{2}\right)$ & 27206,37 & 3806,37 & 14206,37 & 5762,12 & 50981,24 \\
\hline
\end{tabular}

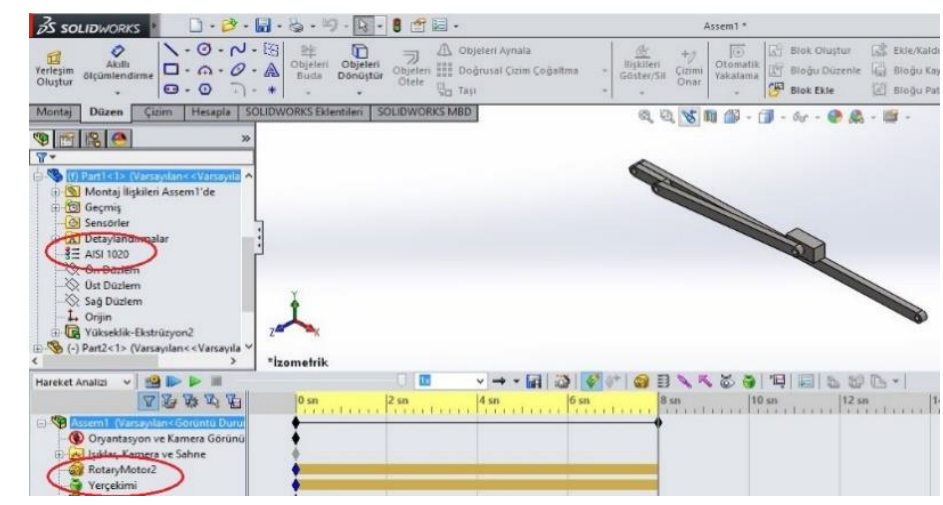

Şekil 2. SOLIDWORKS ekranındaki krank-biyel mekanizması

\section{BULGLAR VE TARTIŞMA}

\subsection{Mekanizmanın Fiziksel Özellikleri}

SOLIDWORKS montajı yapılmış ve malzemesi tanınmış mekanizmamızın kütlesel özelliklerini almamıza yardımcı olmaktadır. Mekanizmanın kütle özelliklerinde olası farkl11ıkları rahatlıkla gözlemleyebilmek içim ölçü birimleri oldukça küçük seçilmiştir. Kütle bilgisini miligram ( $\mathrm{mg}$ ) cinsinden hacim bilgisini nanometre küp $\left(\mathrm{nm}^{3}\right)$ ve yüzey alanın bilgisini nanometre kare $\left(\mathrm{nm}^{2}\right)$ olarak almamızı sağlamaktadır. Tablo 3'de mekanizmanın kütlesel özellikleri verilmiştir. Dosya formatlarına göre elde edilen bilgilerin değişen değerleri, koyu renkte (Bold) ve italik olarak ifade edilmiştir (Tablo 3).

Tablo 3. Krank-biyel mekanizmasının tüm dosya formatları için fiziksel özellikleri

\begin{tabular}{|c|c|c|c|}
\hline & SLDPRT & STEP & IGES \\
\hline Kütle $(\mathrm{mg})$ & 1216684 & 1216684 & 1216684 \\
\hline Hacim $\left(\mathrm{nm}^{3}\right)$ & 15401061929829749 & 15401061929829752 & 15401061929829752 \\
\hline Yüzey alanı $\left(\mathrm{nm}^{2}\right)$ & 50981238596594936 & 50981238596594952 & 50981238596594952 \\
\hline
\end{tabular}

Krank-biyel mekanizmasının her bir uzvunun ayrı olarak ve montajının kaydedilmesi ile oluşan dosya boyutları, IGES, STEP ve SLDPRT, incelenmiştir. Dosya boyutları, Tablo 4 de kilobayt $(1 \mathrm{~Kb}=1024$ Byte $)$ cinsinden gösterilmiştir. 
Tablo 4. Krank-biyel mekanizmasının tüm veri tipleri için dosya boyutları

\begin{tabular}{|c|c|c|c|c|c|}
\hline & Parça 1 (Kb) & Parça 2 (Kb) & Parça 3 (Kb) & Parça 4 (Kb) & Montaj (Kb) \\
\hline SLDPRT & 54 & 69 & 55 & 55 & 398 \\
\hline IGES & 37 & 50 & 39 & 39 & 611 \\
\hline STEP & 40 & 51 & 41 & 42 & 396 \\
\hline
\end{tabular}

Dosya boyutları dikkate alındığında, IGES parça başına en küçük, montaj olarak ise en büyük olduğu görülmektedir. STEP ise IGES ten sonra ikinci sıraya yerleşmektedir. Dosya boyutu, parçaya veya montaja ait tutulan bilginin büyüklüğü olarak değerlendirmesi doğru değildir. Dosya boyutu, kullanılan dosya formatına göre doğrudan değişmektedir. Bununla birlikte, her dosya formatına göre bilginin, saklanma biçimi ve ifade edilmesi değişmektedir.

\subsection{Analizler}

SOLIDWORKS programında, üç farklı dosya uzantısı ile saklanan krank-biyel mekanizması için hareket analizleri ayrı ayrı yapılmıştır Yapılan analizlerde elde edilen tüm grafikler görünüşte aynıdır. Dolayısı ile burada sadece SLDPRT için yapılan analizlerde elde edilen grafikler verilmiştir (Şekil 5).

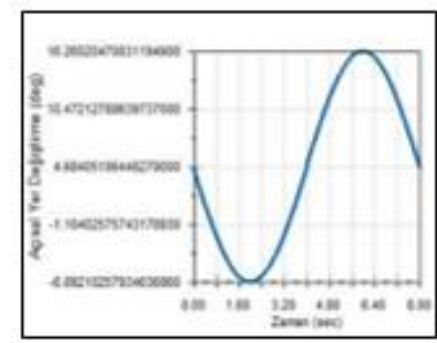

a) Açısal| Yer Değiģtinme

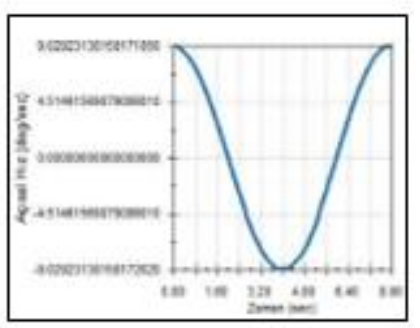

c) Agpral Hiz

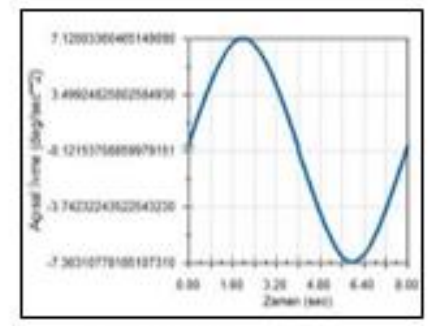

e) Aç1sal İvme

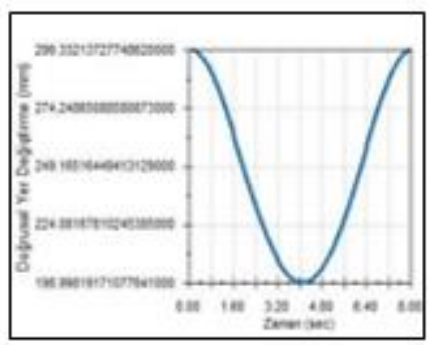

b) Doğrusal Yer Değig̨tirme

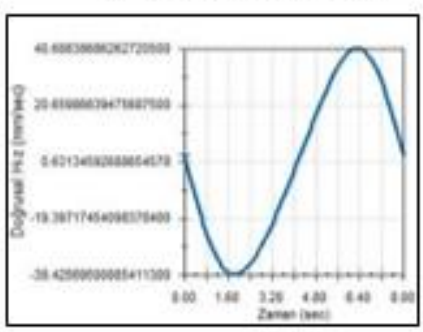

d) Doğrusal Hız

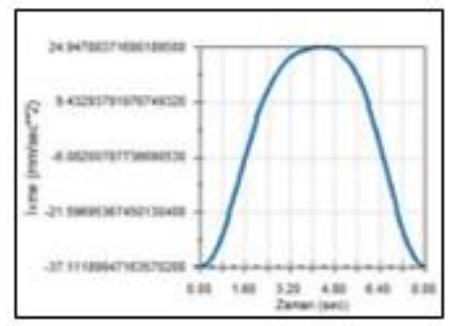

f) Doğnual İvme

Şekil 3. Krank-biyel SLDRT formatı hareket analizi grafikleri 
Verilen grafikler incelendiğinde, zamana bağlı olarak değişen değerler görülmektedir. Grafik değerleri birbirleri ile kıyaslandığında değişimlerin daha rahat anlaşılması için ölçü birimleri (nm, sec, vb.) oldukça küçük seçilmiştir. Dolayısı ile Tablo 5 ve Tablo 6 da verilen değerler, bu grafiklerde çizilen her eğrinin Min. ve Max. noktaları olmaktadır.

Hareket analizinde sonuçlar, üç yönden ele alınmıştır:

A. Konum bilgileri: Açısal yer değiştirme, Doğrusal yer değiştirme,

B. Hız bilgileri: Açısal hız, Doğrusal hız,

C. Ívme bilgileri ise: Açısal ivme, Doğrusal ivme.

Analiz sonuçlarının karşılaştırılmasında tüm verilerin yerine maksimum (Max) ve minimum (Min) değerler dikkate alınmıştır. SOLIDWORKS programında modellenerek oluşturulan krank-biyel mekanizmasına ait veriler karşılaştırmalı olarak Tablo 5 ve Tablo 6 da verilmiştir. İlk olarak Tablo 5'te verilen SLDPRT ile STEP hareket analizi verileri incelendiğinde, sayısal değerlerin birbirinden farklı olduğu görülmüştür. Farklar, virgülden (,) sonra gelen rakamlarda meydana gelmiştir. En büyük farklar, Doğrusal hız değerlerinde yaklaşık $0,1 \mathrm{~mm} / \mathrm{sn}$ oluşmuştur. SLDPRT ile STEP değerleri arasındaki farklar, Tablodaki değerlerin (virgülden sonra) koyu renk ve italik yapılması ile işaretlenmiştir. Tablo 6 verilen SLDPRT ile IGES sayısal değerleri arasında da farklılıkların olduğu görülmüştür. Özellikle farklar:

- Açısal yer değiştirme

- Açısal hız

- Doğrusal hız

- Açısal ivme

Verilerinde oluşmuştur. STEP verilerinde olduğu gibi en büyük fark, Doğrusal hız sonuçlarında yaklaşık $0,1 \mathrm{~mm} / \mathrm{sn}$ meydana gelmiştir. SLDPRT ile IGES değerleri arasındaki farklar, Tablo değerlerin (virgülden sonra) koyu renk ve italik yapılması ile işaretlenmiştir.

Tablo 5. SLDPRT ile STEP için max. ve min. noktalar

\begin{tabular}{|c|c|c|c|c|}
\hline & \multicolumn{2}{|c|}{ SLDPRT } & \multicolumn{2}{c|}{ STEP } \\
\hline $\begin{array}{c}\text { Açısal Yer } \\
\text { değiştirme (Deg) }\end{array}$ & 16,26020470831194900 & $-6,89210257934636860$ & 16,26020474237540400 & $-6,89210261228532420$ \\
\hline $\begin{array}{c}\text { Doğrusal Yer } \\
\text { değiştirme (mm) }\end{array}$ & 299,33213727748620000 & 198,99819171077641000 & 299,33213727748631000 & 198,99819171077652000 \\
\hline $\begin{array}{c}\text { Açısal Hız } \\
(\text { Deg/sn) }\end{array}$ & 9,02923138158171850 & $-9,02923138158172020$ & 9,02923138158171670 & $-9,02923138158171670$ \\
\hline $\begin{array}{c}\text { Doğrusal Hız } \\
(\mathrm{mm} / \mathrm{sn})\end{array}$ & 40,68838686262720500 & $-39,42569500885411300$ & 40,81543539261221600 & $-39,54724063826221700$ \\
\hline $\begin{array}{c}\text { Açılsal İvme } \\
\left(\mathrm{deg} / \mathrm{sn}^{2}\right)\end{array}$ & 7,12003360465149090 & $-7,36310778185107310$ & 7,12003362546465370 & $-7,36310780413990250$ \\
\hline $\begin{array}{c}\text { Doğrusal. İvme } \\
\left(\mathrm{mm} / \mathrm{sn}^{2}\right)\end{array}$ & 24,94788371690189500 & $-37,11189947163570200$ & 24,94788371690189500 & $-37,11189947163570200$ \\
\hline
\end{tabular}


M. Er \& Y. Kayir, Aksaray University Journal of Science and Engineering, 3(1) (2019) 51-60.

Tablo 6. SLDPRT ile IGES için max. ve min. noktalar

\begin{tabular}{|c|c|c|c|c|}
\hline & \multicolumn{2}{|c|}{ SLDPRT } & \multicolumn{2}{|c|}{ IGES } \\
\hline & Max. & Min. & Max. & Min. \\
\hline $\begin{array}{c}\text { Açısal Yer } \\
\text { değiştirme (Deg) }\end{array}$ & 16,26020470831194900 & $-6,89210257934636860$ & 16,26020470831194900 & $-6,89210257934668100$ \\
\hline $\begin{array}{c}\text { Doğrusal Yer } \\
\text { değiştirme (mm) }\end{array}$ & 299,33213727748620000 & 198,99819171077641000 & 299,33213727748620000 & 198,99819171077641000 \\
\hline $\begin{array}{l}\text { Açıssal Hız } \\
\text { (Deg/sn) }\end{array}$ & 9,02923138158171850 & $-9,02923138158172020$ & 9,02923138158172020 & $-9,02923138158172020$ \\
\hline $\begin{array}{l}\text { Doğrusal Hız } \\
(\mathrm{mm} / \mathrm{sn})\end{array}$ & 40,68838686262720500 & $-39,42569500885411300$ & 40,81543539261222300 & $-39,54724063826223100$ \\
\hline $\begin{array}{l}\text { Açısal İvme } \\
\left(\mathrm{deg} / \mathrm{sn}^{2}\right)\end{array}$ & 7,12003360465149090 & $-7,36310778185107310$ & 7,12003360465149090 & $-7,36310778185107660$ \\
\hline $\begin{array}{l}\text { Doğrusal. İvme } \\
\left(\mathrm{mm} / \mathrm{sn}^{2}\right)\end{array}$ & 24,94788371690189500 & $-37,11189947163570200$ & 24,94788371690189500 & $-37,11189947163570200$ \\
\hline
\end{tabular}

Tablo 5 ve Tablo 6'da verilen Max. ve Min. değerlerinden Tablo 7'de verilen yeni değerler elde edilmiştir. Tablo 7'de verilen değerler, Tablo 5 ve Tablo 6'daki Max. ve Min. değerlerin birbirinden çıkarılması (fark) ile elde edilmiştir. Burada amaç, SOLIDWORKS programının kendi kullandığı SLDPRT dosya uzantılı parçalardan elde edilen Krank-Biyel mekanizması ile diğer dosya uzantılı (IGES ve STEP) parçalarla oluşturulan Krank-Biyel mekanizması analiz değerlerini karşılaştırmak, hata miktarlarını belirlemektir.

Tablo 7'de SLDPRT analizi değerlerine en yakın sonuçları veren dosya formatının IGES olduğu görülmektedir. Elde edilen 0 (sıfır) değerleri bu durumu ortaya koymaktadır.

Tablo 7. Elde edilen max. ve min. değerleri arasındaki fark

\begin{tabular}{|c|c|c|c|c|}
\hline & \multicolumn{2}{|c|}{ SLDPRT- STEP } & \multicolumn{2}{c|}{ SLDPRT- IGES } \\
\hline & Max. Hata & Min. Hata & Max. Hata & Min. Hata \\
\hline $\begin{array}{c}\text { Açısal Yer } \\
\text { değiştirme (Deg) }\end{array}$ & $-0,00000003406345500$ & $-0,00000003293895560$ & $\mathbf{0}$ & $-0,00000000000031240$ \\
\hline $\begin{array}{c}\text { Doğrusal Yer } \\
\text { değ }\end{array}$ & $-0,000000000000011000$ & $-0,000000000000011000$ & $\mathbf{0}$ & $\mathbf{0}$ \\
\hline $\begin{array}{c}\text { Açırıal Hız } \\
(\text { Deg/sn) }\end{array}$ & 0,0000000000000180 & 0,00000000000000350 & $-0,0000000000000170$ & $\mathbf{0}$ \\
\hline $\begin{array}{c}\text { Doğrusal Hız } \\
(\mathrm{mm} / \mathrm{sn})\end{array}$ & $-0,127048529985011$ & $-0,121545629408104$ & $-0,0127048529985018$ & 0,121545629408118 \\
\hline $\begin{array}{c}\text { Açısal İvme } \\
\left(\mathrm{deg} / \mathrm{sn}^{2}\right)\end{array}$ & $-0,0000002081316280$ & $-0,00000002228882940$ & $\mathbf{0}$ & $-0,00000000000000350$ \\
\hline $\begin{array}{c}\text { Doğrusal. İvme } \\
\left(\mathrm{mm} / \mathrm{sn}^{2}\right)\end{array}$ & $\mathbf{0}$ & $\mathbf{0}$ & $\mathbf{0}$ & $\mathbf{0}$ \\
\hline
\end{tabular}

\section{SONUÇLAR}

$\mathrm{Bu}$ çalışmada SLDPRT, STEP ve IGES dosya formatında kayıt altına alınan parçalardan oluşturulan bir krank-biyel mekanizmasının SOLIDWORKS ortamında hareket analizleri 
yapılmıştır. Mekanizma parçalarının SLDPRT, STEP ve IGES formatlarında kaydedilmesi ile bilginin dönüştürüldüğü anlaşılmıştır. Dolayısıyla bu dönüşümlerde veri kayıplarının meydana geldiği ortaya çıkmıştır. Modellenen Krank-Biyel mekanizmasının SLDPRT, IGES ve STEP dosya formatlarında saklanması ile fiziksel bilgilerinin (Yüzey alanı, hacim) değiştiği belirlenmiştir. Krank-Biyel mekanizması parçalarının SLDPRT, STEP ve IGES dosya formatlarında kaydedilmesi ile elde edilen dosya boyutları da $(\mathrm{Kb})$ değişmiştir. Büyüklük sırasına göre dosya boyutları, SLDPRT, IGES ve STEP şekilde meydana gelmiştir. Yapılan hareket analizi sonuçlarının birbirinden farklı olması tespit edilen bu farklılıklardan (kayıplardan) kaynaklandığı düşünülmektedir. Bu kayıpların daha karmaşık parçalarda daha fazla olacağı anlaşılmaktadır. Analiz sonuçları dikkate alındığında SLDPRT’e en yakın sonuçların IGES ile ulaşıldığı tespit dilmiştir. Dolayısı ile SOLIDWORKS programında yapılacak olan hareket analizlerinde, dişarıdan ihtiyaç duyulan parçaların IGES olarak alınmasının daha doğru olacağı anlaşılmaktadır.

\section{Kaynaklar}

[1] A. Çiçek, Sakarya Üniversitesi Fen Bilimleri Enstitüsü Dergisi, 10 (2) (2006) 31-38.

[2] S. Marjudi, M.M. Amran, K.A., Abdullah, S. Widyarto, N.A. Majid, R. Sulaiman, Proceedings Of World Academy of Science, Engineering And Technology, (62) (2010) 1013-1017.

[3] M. Zapciu, D. Anania, D. Tilina, Scientific Bulletin Series C: Fascicle Mechanics, Tribology, Machine Manufacturing Technology, (20) (2006) 383.

[4] Z. Tekiner, İ. Korkut, Turkish Journal of Engineering and Environmental Sciences, 25 (1) (2001) 31-38.

[5] M. Gülesin, Y. Kayır, Teknoloji, 8 (2) (2005) 123-137.

[6] M.P. Bhandarkar, B. Downie, M. Hardwick, R. Nagi, Computers in Industry, 41 (2000) 261-277.

[7] İ. Çelik, S. Yaldız, A. Ünüvar, Dumlupınar Üniversitesi Fen Bilimleri Enstitüsü Dergisi, (9) (2005) 139-155.

[8] K.M. Hu, B. Wang, Y. Liu, J. Huang, J.H. Yong, .The International Journal of Advanced Manufacturing Technology, 58(9-12) (2012) 1155-1170.

[9] E. Söylemez, Mekanizma tekniği, Dördüncü baskı (Birsen Yayınevi, İstanbul, 2017) s.324.

[10] https://cadsay.com/SOLIDWORKS-nedir? (2019). 